\title{
Potential Opportunities for Development of Mechanical Engineering with The Context of Historical Evolution of This Engineering Branch
}

\author{
Jacek Jackiewicz* \\ Faculty of Mechatronics, Kazimierz Wielki University in Bydgoszcz, Poland \\ *Corresponding author: Jacek Jackiewicz, faculty of Mechatronics, Kazimierz Wielki University in Bydgoszcz, Poland
}

\section{Short Communication}

Mechanical engineering is not only one of the oldest engineering disciplines but also the broadest one, i.e., with the wide-scope as well as a large number of its branches. This engineering discipline requires a clear understanding of the principles of engineering physics, engineering mathematics, and materials science to design, analyze, manufacture, and maintain mechanical systems such as mechanisms, devices, and machines.

With a view to machines' development, the progress of mechanical engineering advancement is often directly related to the course of civilization history. Therefore, it can be studied from many perspectives. Our attention often directs to significant particular forms or stages of civilization, which arisen throughout human history. One cannot forget about ancient cultures, both Greco-Roman (in Europe) and Chinese (in Asia), which adopted to a great extent and, then, developed often anonymous technical achievements of the first civilizations of the prehistoric period [1].

The illustrious ancient achievements had been followed by the technical culture of Islam, which not only systematized them but also improved a bit afterward. In the late Middle Ages, these achievements spurred European societies to search for new mechanical devices and, thus, the foundations were laid for the subsequent Renaissance. During the Renaissance and also after it, there was an excellent development of creative and innovative engineering thinking enshrined in treaties on machines, especially in the anthology of Italian, French as well as German technical literature. The Industrial Revolution was the culmination of all previous technological advances and which manifested itself by the transition of production processes from old manual methods to new ones supported by machines.
Mechanical engineering may be treated as a precursor to many branches of engineering, including mechatronics that is an interdisciplinary branch of mechanical engineering and electronic engineering at least, to create controlled hybrid systems, thanks to which machines can be easily automated. Besides, the use of microprocessor-based digital embedded controllers, together with a wide selection of control software algorithms dedicated to these programmable microcontrollers, enables us to build specific pieces of automation equipment based on electric motors, servomechanisms, and other electrical systems. Mechatronic structures are the result of the evolution of mechanical objects, bearing in mind the development history of mechanical engineering. According to the conceptual block diagram shown in Fig. 1, the application of digital technologies in mechatronic systems has provided a very different approach to automatic control [2,3]. Thanks to the use of modern digital computers, more and more often, in the case of advanced control methods, purely mathematic models of systems' behavior and controllers' algorithms are abandoned in favor of sophisticated artificial intelligence (AI) techniques with the help of which control systems can learn about their environment and properly modify their behavior in conjunction with control based on knowledge to ensure their optimal performance. For this to be possible, advanced control systems must have sufficient computational capacity to create not only representative mathematical models of the controlled systems but also to modify their own adaptive control actions [4].

Derived from Babbage and Lovelace's analytical engine digital computers with silicon chips of advanced mechatronic systems are now an inspiration to build biological computers [5] for massive 
computing tasks. In these machines, each processor consists of a single biological macromolecule. By employing a significantly large number of such macromolecules in parallel systems, solving computational problems will be run more quickly than using the contemporary fastest conventional supercomputers. The application of biological computers may also be relevant from another point of view. Namely, advances in the construction of intelligent materials can cause the stage for the disappearance of physical segregation between sensors, actuators, and controllers in micro-electromechanical systems (MEMS). Moreover, note that this is beneficial because intelligent materials used in MEMS devices convert electrical or chemical energy directly to mechanical energy through adequate responses of such kind of materials.

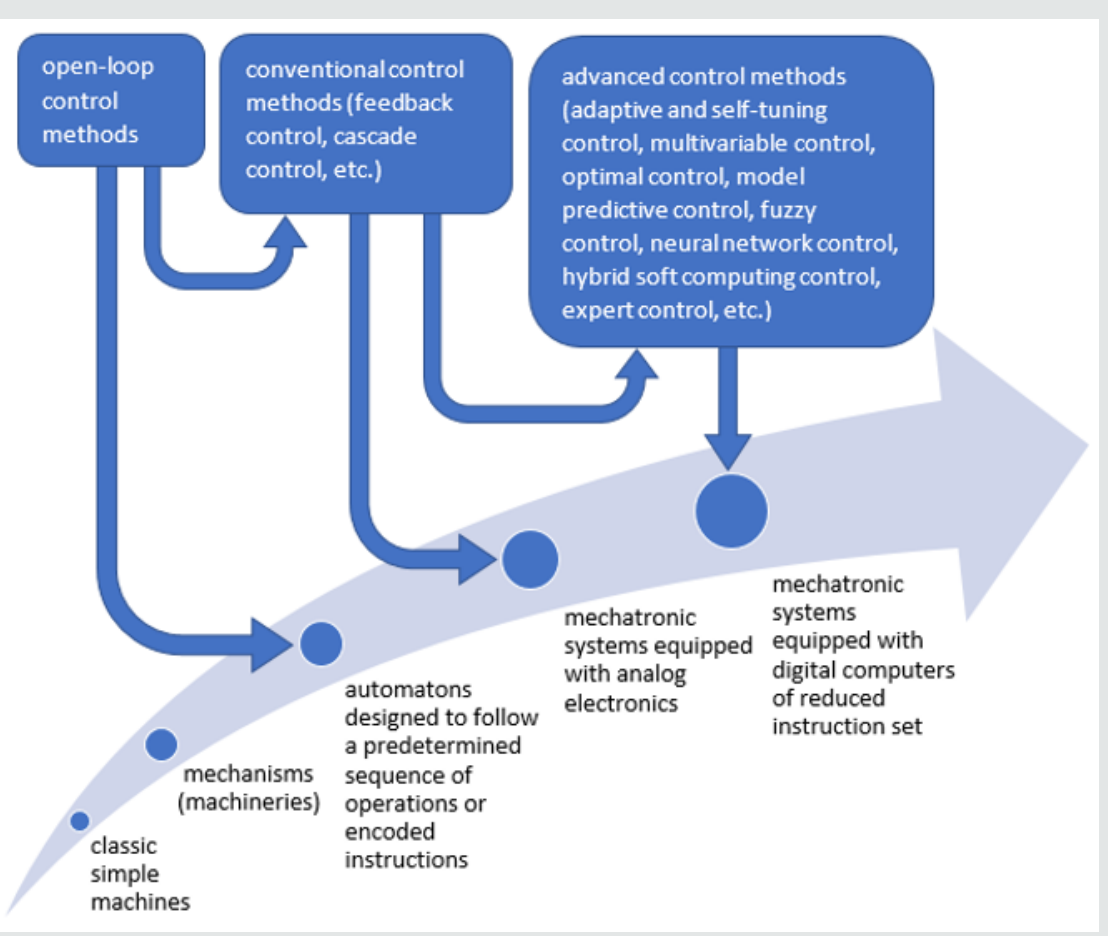

Figure 1: Mechatronic structures as the result of the evolution of mechanical objects.

As a result of the comparison of the crucial turning points in human history with the dynamics of machines' development, parallel evolutionary processes can be perceived, which are reflected in the periods of both political and economic hegemonies of the most technologically advanced factual civilizations. Nevertheless, the history of mankind tells us also that some policymakers supported successfully uncivilized methods of domination exemplified by the Opium Wars, which were waged between representatives of the China Qing dynasty and the Kingdom of Great Britain supported by other European countries. In the $18^{\text {th }}$ and $19^{\text {th }}$ centuries, there was a strong request for Chinese luxury goods as tea, silks, and porcelain pottery, for which Europeans had to pay with gold or silver or automaton clocks and pocket watches as the Qing emperors developed an interest in these mechanical curiosities. However, the offer of the European market at that time was insufficient to reduce the deepening trade imbalance of European countries with China. Therefore, the Kingdom of Great Britain, mainly, started exporting opium grown in India and sold it to China.

However, it is reassuring that new scientific achievements make it possible today to do what just yesterday seemed impossible. It is comforting that even if these are military achievements, some of them can be utilized for the benefit of humanity. For example, instead of precise bombing by means of homing missiles with grid fins [6], Elon Musk from SpaceX used such type of missile guidance system to partially reusable launch systems Falcon 9 of which the first stage having the ability to re-enter the atmosphere and landing back vertically after separating from the second stage.

\section{Conclusion and future directions}

Just a few years ago, it seemed as though it would be many more years for AI developed enough to threaten human creativity. However, we are moving towards a situation where artificial intelligence in particular areas of application will start to match human intelligence or even become much smarter than humans. This intelligence is especially useful when designing flexible and intelligent mechatronic systems with adaptive controllers that can be reconfigured both in hardware and software to suit specific tasks. Such control systems, with reconfigurable multiprocessors, quite often use the field-programmable gate array (FPGA) technology [7] introduced at the beginning of the 80 s of the twentieth century. Anyone of the FPGA integrated circuit, which contains an array 
of programmable logic blocks and a hierarchy of reconfigurable interconnects, can be used to build a computer.

In modern times of the use of nanometer-scale chips, due to the predictable and resulting from Moore's law limitations of silicon computing devices achieving the ultra-dense scale of integration, people began to think about the application of molecules to solve computational problems. Molecular logic combinational gates can be implemented as a molecular calculator, which can perform various arithmetic operations, specifically, addition and subtraction [8]. Inspired by the ideas of digital electronic computing based on Boolean functions, the DNA computing technique performs calculations using biological molecules and can be realized by multi-layered circuits [9]. Moreover, using ribonucleic acid (RNA), which is a crucial molecule with long chains of nucleotides scientists have vindicated that living cells can be induced to carry out complex computations, which are carried out in a similar way as by computers or controllers of tiny robots. Thus, these living cells make 'living computers'.

Considering that 100,000 neurons in a piece of brain matter the size of a grain of sand, the computational capabilities of biological computing are practically endless. It is evidenced by the statement provided in [10], viz 100 thousand neurons enable robotics with multiple sensory inputs. However, 1 million neurons will make possible the creation of a computer, which can think for itself.

Besides, this technology will make it easier to create mobile microrobots for bioengineering applications.

\section{References}

1. Sanz JLM, Paz EB, Ceccarelli M, Otero JE, Morgado PL, et.al.(2009) The Evolution and Development of Mechanical Engineering Through Large Cultural Areas. In International Symposium on History of Machines and Mechanisms (pp. 69-82).

2. Bennett S (1996) A brief history of automatic control. IEEE Control Systems Magazine, 16(3): 17-25.

3. Kozak S (2014). State-of-the-art in control engineering. Journal of Electrical Systems and Information Technology, 1(1): 1-9.

4. Bissell C (2009) A history of automatic control. In Springer handbook of automation (pp. 53-69)

5. Moe-Behrens GH (2013) The biological microprocessor, or how to build a computer with biological parts. Computational and structural biotechnology journal, $7(8)$.

6. Reynier P, Longo JM, \& Schulein E (2006) Simulation of missiles with grid fins using an actuator disk. Journal of spacecraft and rockets, 43(1): 84-91.

7. Kelly JS, Rao VS, Pottinger HJ, \& Bowman HC (1997). Design and implementation of digital controllers for smart structures using field programmable gate arrays. Smart materials and structures, 6(5): 559.

8. Erbas-Cakmak S, Kolemen S, Sedgwick AC, Gunnlaugsson T, James TD,et. al. (2018). Molecular logic gates: the past present and future. Chemical Society Reviews, 47(7): 2228-2248.

9. Qiu M, Khisamutdinov E, Zhao Z, Pan C, Choi JW, et.al. (2013). RNA nanotechnology for computer design and in vivo computation. Philosophical Transactions of the Royal Society A: Mathematical, Physical and Engineering Sciences, 371(2000): 20120310.

10. McShane S (2016). This Amazing Computer Chip Is Made of Live Brain Cells. https://singularityhub.com/2016/03/17/this-amazingcomputer-chip-is-made-of-live-brain-cells/

11. Ceylan H, Giltinan J, Kozielski K, \& Sitti M (2017). Mobile microrobots for bioengineering applications. Lab on a Chip, 17(10), 1705-1724. (c) $\left(\begin{array}{l}\text { This work is licensed under Creative } \\ \text { Commons Attribution 4.0 License }\end{array}\right.$

To Submit Your Article Click Here: Submit Article

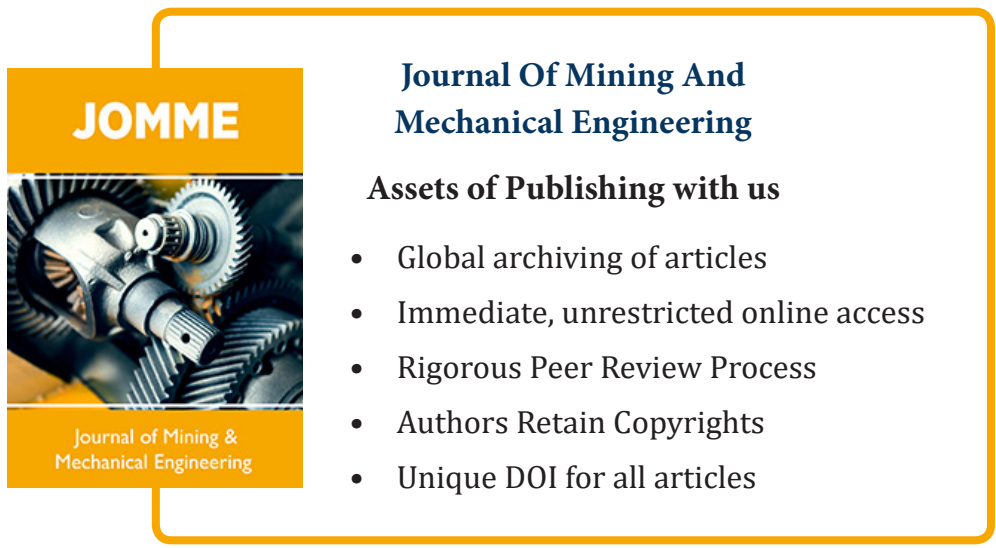

Citation: Jacek Jackiewicz. Potential Opportunities for Development of Mechanical Engineering with The Context of Historical Evolution 